\title{
Implementation of a Two-Level Security Door Access Using Keypad and Voice Recognition
}

\author{
Morkat M. Yilwatda $^{1 *}$, Jonathan A. Enokela ${ }^{2}$ and Nentawe Y. Goshwe ${ }^{3}$ \\ 1, 2, ${ }^{3}$ Department of Electrical and Electronics Engineering, \\ University of Agriculture, P.M.B. 2373, Makurdi, Benue State, Nigeria \\ ${ }^{1}$ Tel: +2347031884040 \\ 'manmorkat@gmail.com, ${ }^{2}$ jonajapeno@gmail.com, ${ }^{3}$ nentawe@gmail.com
}

\begin{abstract}
It is possible today to automate a growing number of speaker-recognition tasks with such technologies as voice or speaker verification and voice identification. These systems have been applied to real-world security applications. They have, however, been undergoing modifications due to the poor level of security architecture that they provide to users. Hence there is the need, as has been done in this work, to develop a more efficient, user friendly and complex security system that can curtail illegal intrusion into special facilities. The system described in this paper uses matrix keypad which is interfaced with a microcontroller for first level security validation to monitor and control the execution of desired tasks within the keypad and voice recognition (KVR) system. A voice recognition Integrated Circuit is used for second level security verification. A tristate buffer is also employed to logically isolate the buses of the digital signal processing (DSP) chip and those of the microcontroller. From the research, tests were conducted for both noiseless and noisy environments and the recommended distance between the user and microphone in a noiseless environment using electrets type condenser microphone is $1.0 \mathrm{~cm}$ to $16 \mathrm{~cm}$ while under noisy condition it was found to be $1.0 \mathrm{~cm}$ to $6.0 \mathrm{~cm}$. These results depend on the sensitivity of the microphone and the environmental conditions at the time. It can be deduced from the research that integrating the keypad and voice recognition design can help to optimize the security level of the system and by implication help in controlling unwanted intrusion into the special facilities such as museum, military armory, financial institution, etc.
\end{abstract}

Keywords: Voice recognition, access control, electronics security

\section{Introduction}

Contemporary events around the world emphasize the importance of security to lives and properties. According to Organization for Economic Cooperation and Development, security is fundamental to reducing poverty, protecting human rights and creating an enabling environment for development [1]. The concept of security for protection of doors has been broadened beyond the traditional notions of lock and key method to include the use of microcontroller based digital lock for safety of valuables.

Voice synthesis is among the microcontroller based security architecture used for protection of doors and valuables. It is mainly divided into two parts: voice or speaker dependent (SD) and speaker independent (SI) voice recognition [2]. SD voice recognition systems are trained by the person who will be using the systems and these systems respond accurately only to the person who trained them. SI systems are trained to respond to words independent of the user. As a result of this the accuracy of such systems is much lower and their vocabularies are also rather limited compared to SD systems. Most

${ }^{*}$ Corresponding Author 
commercially available speaker recognition devices are general purpose SI systems. Voice biometrics, like other forms of biometrics technology, is used in the security system because it has a higher security level which cannot be stolen, created, forgotten, shared or lost [3]. These unique characteristics show distinctiveness which means each person has difference enough to distinguish him based on these characteristics. Using these characteristics, a robust two-level security door access using keypad and voice recognition (KVR) methods has been implemented.

\section{Literature Review}

There are some projects that have been completed with regards to the application of voice as the controlling method. Krishna and Kranti [4] proposed the design and implementation of a microcontroller based voice controlled wheelchair for handicapped patients. The patients who cannot walk and have to use a wheel chair can steer the wheel chair with their voices. The design uses one input device which is the microphone to take input from the user in the form of speech which is then processed by the voice recognition chip HM2007 [5]. On receiving the signal the microcontroller directs the motors through the control circuit. Two DC high torque stepper motors are used for controlling the two wheels of the chair independently. These stepper motors are very useful in rotating the wheelchair at particular angles. If a user wishes to rotate the motor at $30^{\circ}, 45^{\circ}, 60^{\circ}$ he would have to give a command to the motor through a simple program and the motor shaft will move to that particular angle. One of the major setbacks of this design is the cost of maintaining the system. For the system to be mobile the power cells would have to be maintained frequently. Wahyudi and Syazilawati [6] proposed an Intelligent VoiceBased Door Access Control System Using Adaptive-Network-based Fuzzy Inference Systems (ANFIS) for building security that uses a personal computer (PC) equipped with sound card for speaker verification. The sound card records the voice data based on the sampling frequency of $22 \mathrm{kHz}$. In this system, all of the voice data processing and speaker verification algorithms are implemented in the PC using MATLAB and its toolboxes. As a result of the voice-based verification, a decision signal which will accept or reject the access will be sent through the parallel port of the PC to the door access control. The accuracy of the access control system in this design was specified based on the rate at which the system makes decision to reject the authorized person and to accept the unauthorized person.

According to Dhawan and Aditi[7]a single portable unit which can allow one to wirelessly control lights, fans, air conditioners, television sets, security cameras, electronic doors, computer systems, audio/visual equipment, etc, and turn on or off any appliance that is plugged into a wall outlet, get the status of different sensors and take decision accordingly, can be made possible using the Voice Recognition Wireless Home Automation System Based on Zigbee. The overall system is controlled from a microphone which is connected to the HM2007 speech recognition chip. This chip sends the voice commands in binary sequence to a microcontroller. The base station unit takes decision and sends the commands to a remote station. The remote system receives the commands through ZigBee transceiver and performs the requested function.

Omar et al. [8] proposed a new efficient home automation security based on multimodal biometrics. The multimodal biometrics is a combination of different biometric modalities into a single system. The proposed system used the fingerprint and voice as biometrics features for authentication to access control to the home. The system consists of two parts: the first part is the hardware system components and the second part is the proposed system method. The hardware section includes the Arduino Galileo from Intel, fingerprint shield, EasyVR 2.0 voice recognition shield, and GPRS/GSM shield. The design was aimed at increasing the security access control by using multimodal biometrics. 
In a related research Yahya [2] designed a calculator using the HM2007 voice recognition system. The voice-activated calculator was able to recognize spoken words in natural environments, and was able to run the recognition code on it. The resulting output was displayed on the LCD in real-time, and the calculations performed by the microcontroller were also carried out in real-time. However, the major obstacle to successful implementation of the recognition system of this design was the HM2007 itself. Due to the memory restrictions, the size of the training codebook could not be made to be large enough. The system performed most of its functions, although it did not work as a whole.

The system in this project provides a more efficient and reliable voice activated switching device with an output to control the switching of a DC motor and a keypad to provide password for user authentication by integrating the HM2007 voice recognition device with the microcontroller and the keypad password verification. With proper experiment involving training, this voice activated switching system can be used in daily life.

\section{Materials and Methods}

\subsection{Hardware and Software Development Processes}

The design criteria for the Keypad and Voice Recognition (KVR) Fusion in Security Door were defined by the capabilities of the device to recognize a particular voice pattern and execute the desired task. The KVR system is divided into two main components: A voice recognition component, and a microcontroller core component. The microphone, voice recognition integrated circuit (HM2007), and the SRAM constitute the voice recognition components while the LCD, keypad, motor, buzzer and PIC16F877A constitute the microcontroller components. The control unit handles the communication between the voice recognition and user interface. The microcontroller is programmed using the $\mathrm{C}$ programming language through the CCS compiler [9].

\subsection{Block Diagram of the System}

The block diagram of the system is given in Figure 1.

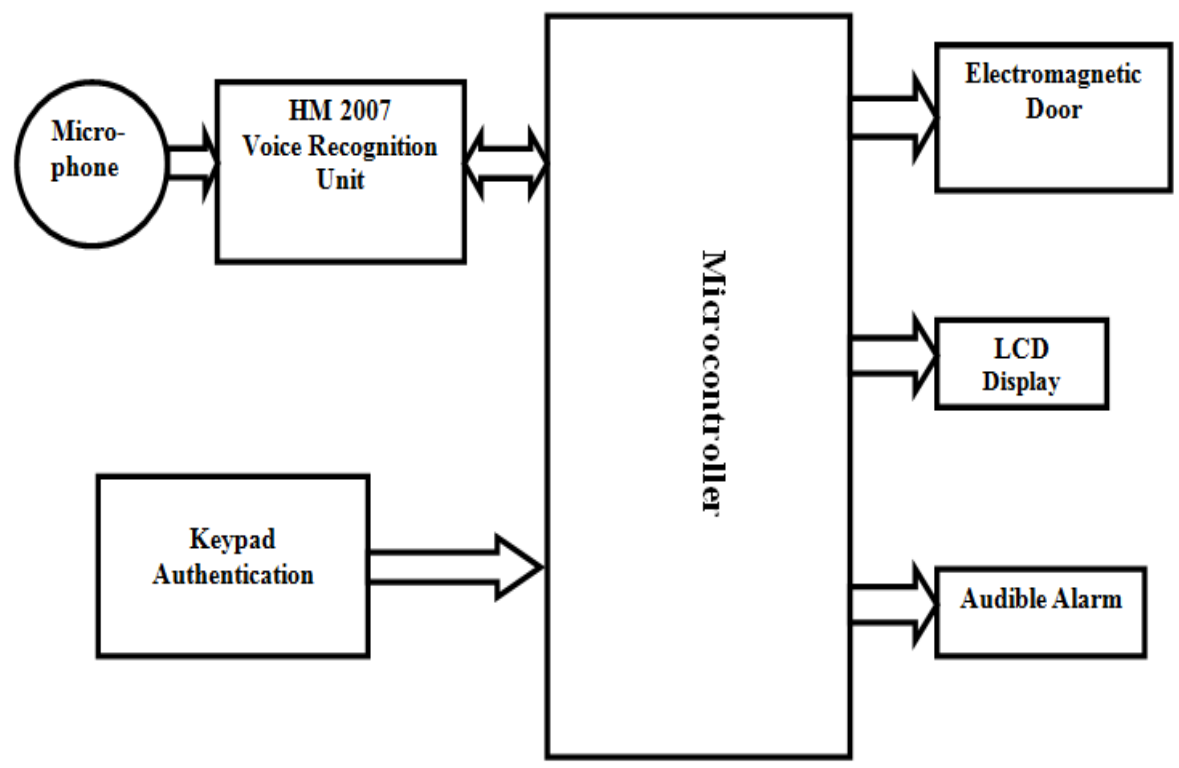

Figure 1. Block Diagram of KVR Security Door 


\subsection{Block Diagram Details and Functions}

\subsubsection{Keypad Select Module}

It is an input device designed to issue instruction to the microcontroller. Each key, upon being depressed, transmits its instruction to the central processing unit of the microcontroller. The keypad used is a $3 \times 4$ matrix keypad. It has 12 buttons in total: $0-9$, * and \#; it is only used to enter the password that allows access to the system and hence, provides the first security clearance for a user. It is also used during the training process of the voice recognition system. The $*$ and \# keys are used to access the program menu and other special functions. Each of the buttons is tied to a $4.7 \mathrm{k} \Omega$ pull-up resistor.

\subsubsection{HM2007 Voice Recognition Unit}

The voice recognition system HM2007 chip is a completely assembled and easy to use programmable speaker recognition circuit. The chip has the basic function of analyzing and comparing an input voice sample with a database of pre-recorded samples. It accomplishes this through an in-built Artificial Neural Network (ANN) model. Each time a user says a word, the chip integrates this word into a neural network and later, in recognition mode, it tries to match the spoken word against other words in its neural net. If a match is made, the index of that word in the vocabulary is returned. If no match is found, or if the user spoke too quickly or too slowly, an appropriate error code is returned.HM2007 provides the option of recognizing either 40 words each with a length of 0.96 second or 20 words each with a length of 1.92 seconds. It does not have an internal memory. It is thus connected to an external $8 \mathrm{k} \times 8$ SRAM memory with 13 address lines. It also has backup memory battery for the SRAM. This battery keeps the trained words safely stored in the SRAM when the main power is turned off. The HM2007 and its connections to other components are shown in Figure 2.

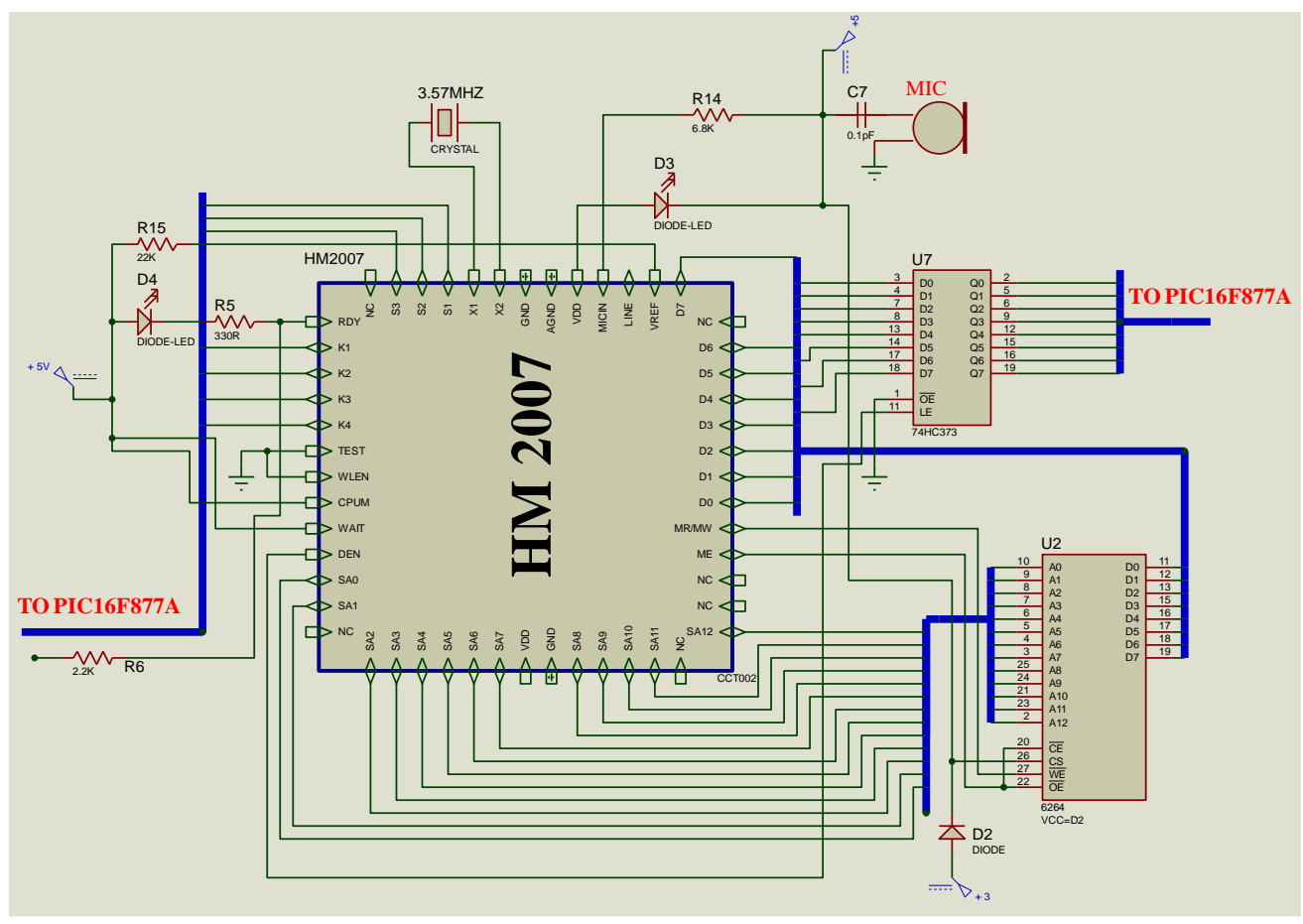

Figure 2. HM2007 Architecture 


\subsubsection{Octal Tri-State Buffer '74LS244' and Octal Transparent Latch '74LS373'}

The tri-state buffer is used in this design to enable a logical isolation between the $\mathrm{K}$ bus and S-bus of the HM2007 and the Port A 'pins 1-4', Port E 'pins 0-2' of the PIC16F887A Microcontroller, since multiple signals will be flowing through these busses during the security code verification and training process of the voice recognition chip. The data out of the HM2007 are clocked into the latch. The outputs of the latch are enabled when the enable control line goes low. The digital binary output from the latch is used to carry out control of the motor through the DC motor driver.

\subsubsection{The Microcontroller Chip}

Since the project's focus is on embedded hardware and software control, the microcontroller is the heart of the system. The microcontroller selected for this project reads digital values from its input/output ports and displays them on the LCD. For the implementation of the circuit functions, the processing capabilities of a PIC16F877A were found to be suitable. It is a fairly large 40-pin microcontroller [10]. It is chosen because a minimum of three full 8-bit ports are required. This also gives the option to increase the number and kind of tasks used in the system.

\subsubsection{Interfacing Microcontroller with other Peripherals}

The microcontroller also drives a dc motor through the L293D driver IC [11]. A liquid crystal display (LCD) gives the visual indications of the processes of the system while a buzzer is used as an audible signaling device. These are connected as shown in Figure 3.

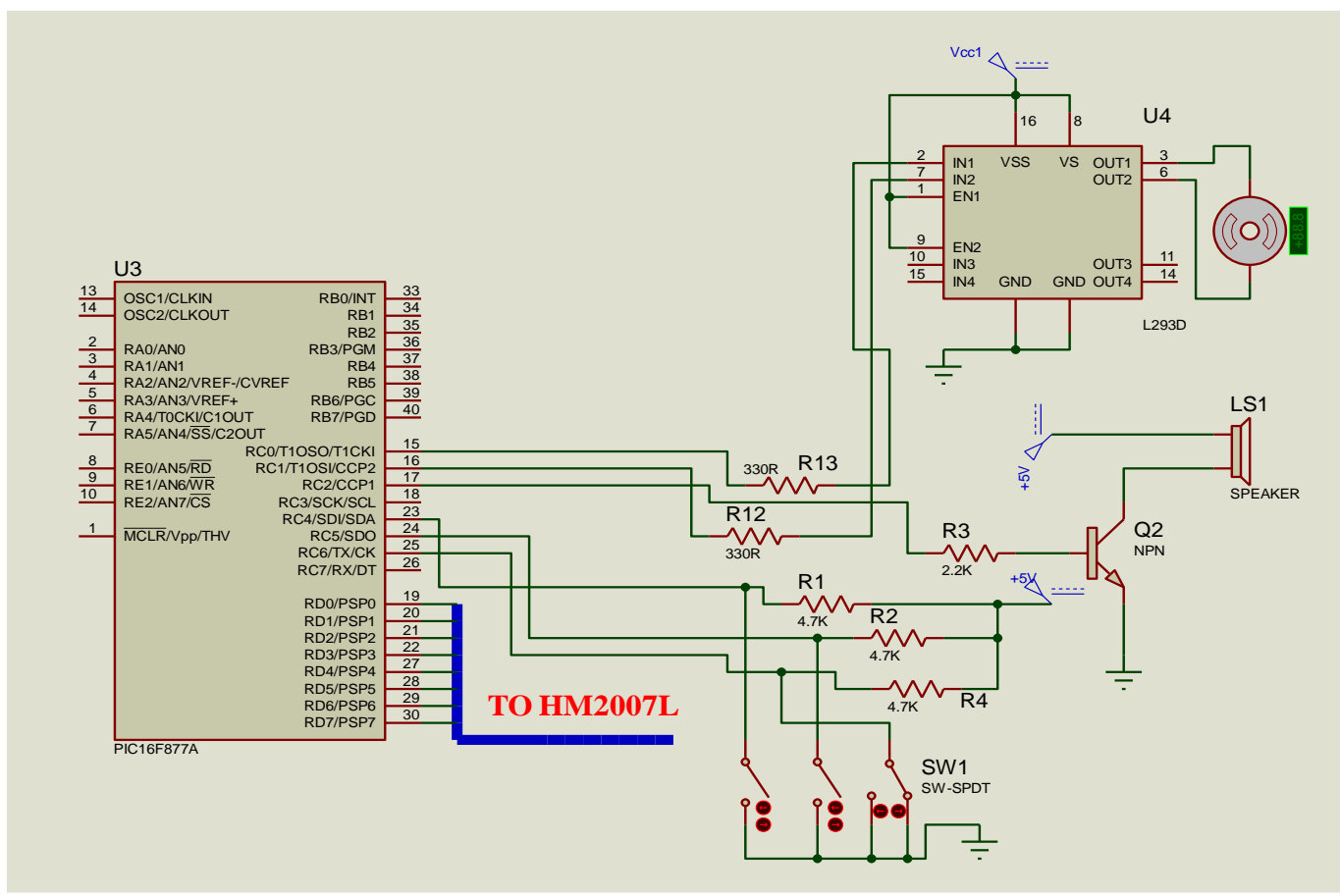

Figure 3. Interfacing Microcontroller with Motor Driver IC

\subsection{System Hardware Interactions and Interconnections}

The circuit operates in the HM2007's manual mode. This mode uses a simple keypad and digital display to communicate with as well as to program the DSP chip; but this cannot be achieved without the special intervention of the microcontroller. The microcontroller therefore, monitors and controls all the activities within the KVR system. 
First, when the circuit is turned on, the HM2007 runs a quick check on the static RAM for error. If no error is found, the RDY pin will be turned active low indicating that the IC is ready for training purpose; this will be sensed by the MCU followed by a corresponding display on the LCD.

After the initialization process, voice inputs can be fed through the microphone that is connected to the MIC_IN pin of the IC. The keyboard as used here has dual functions: authentication and training purpose. There is, therefore, the need to separate the transmission of data through the K-bus and S-bus of the DSP chip during the authentication stage and the training stage. The tri-state buffer '74LS244' IC is employed for this purpose. At the time of training or storing voice input as commands to the system, a 13-bit address is passed onto the SRAM memory, where the addresses are defined, containing information about what commands are stored and where in the memory. The interfaced keypad is used to provide number input corresponding to each word when training the system. Later, during the recognition process, commands are spoken into the microphone, an 8-bit data is sent to the SRAM from the HM2007 and the SRAM matches the command for the address where it is stored. If the input data of the command does not match the stored commands, then an error signal is generated; otherwise the command is passed onto the latch IC '74LS373'. PORT-B of the Microcontroller is interfaced with the LCD and is programmed such that the corresponding word is displayed on the LCD. When there is a match during the recognition process, the HM2007 sends the result to the microcontroller through the latch which will, in turn, send a high signal to the output pins RC0 and RC1 to drive the DC motor through the driver IC L293D, which opens and closes the door. Figure 4 shows the complete hardware design of the voice recognition system while Figure 5 shows the interconnection of the physical hardware.

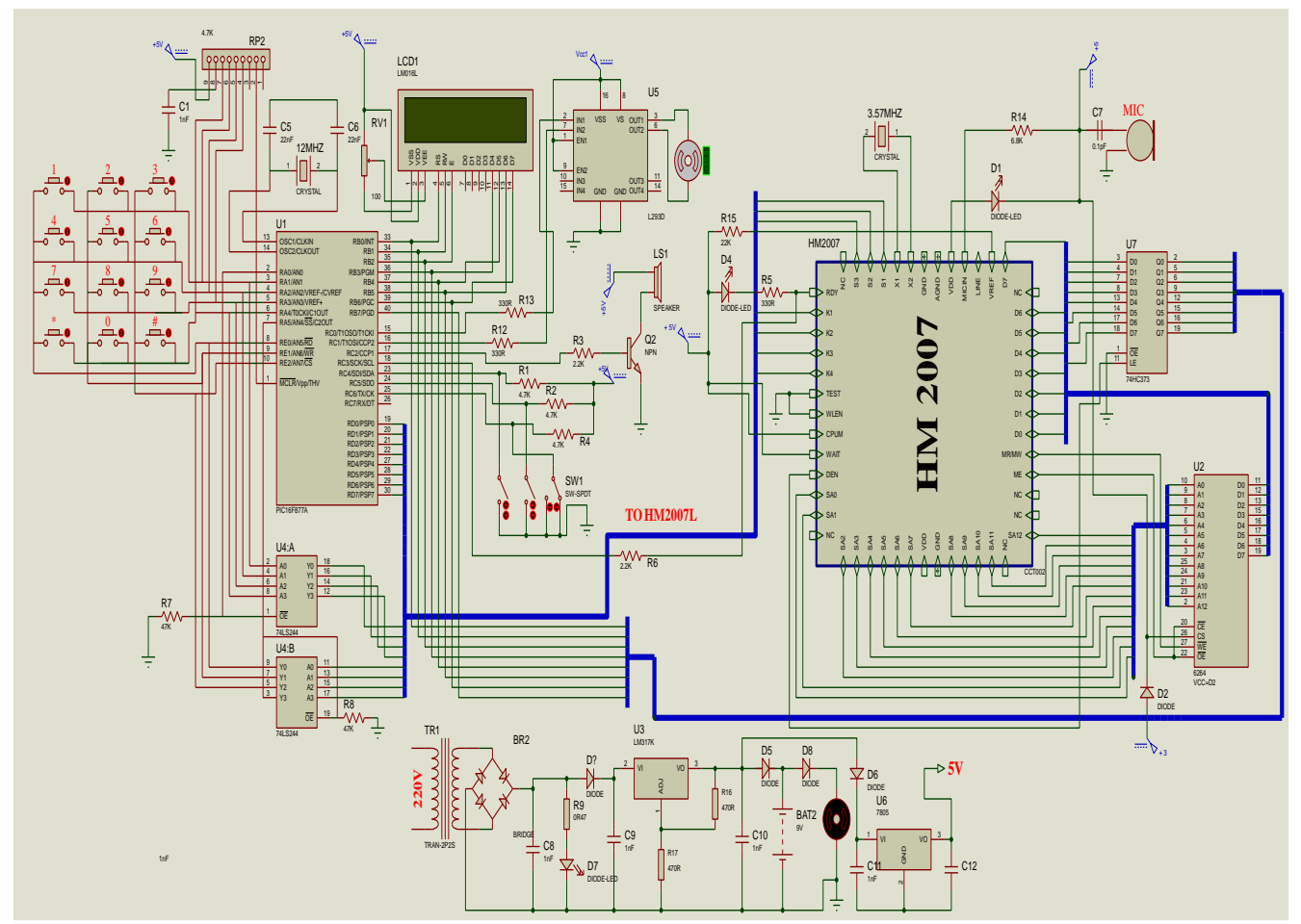

Figure 4. Schematic of the Complete Hardware Interconnection of the KVR 


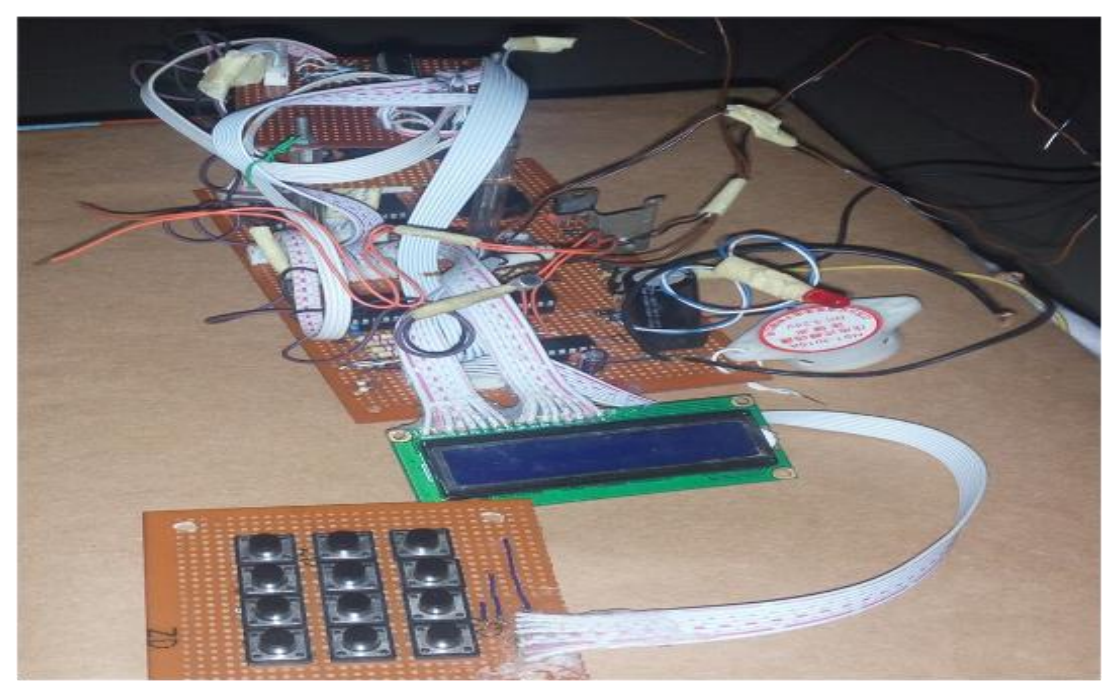

Figure 5. Hardware Interconnection of the KVR

\subsection{System's Flowchart}

The flowcharts showing the composition of the system's software are shown in Figure 6 and Figure 7. The codes corresponding to these flowcharts were written in C programming language using the CCS compiler. The resultant hex file was burnt into the memory of the microcontroller using the PIC kit2 programmer [12].

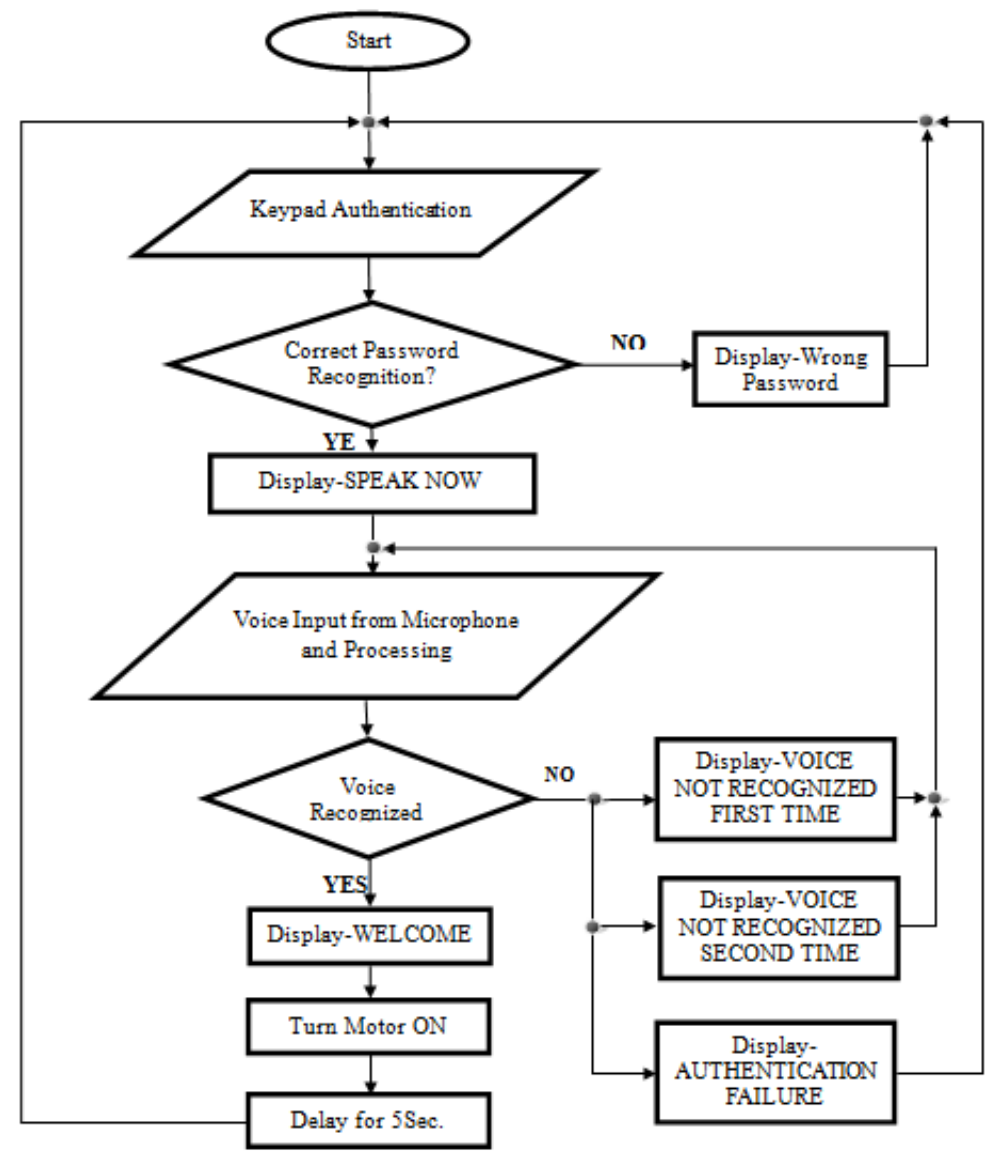

Figure 6. Flowchart of the Keypad and Voice Recognition Software 


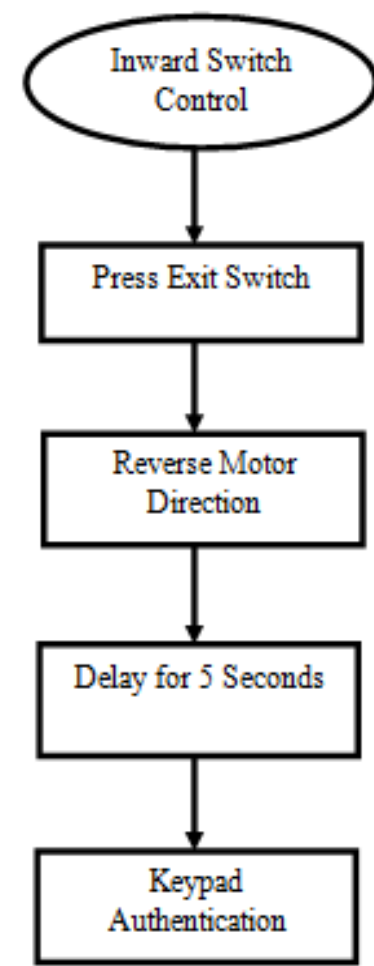

Figure 7. Flowchart for Inward Switch Control Software

\section{Results}

\subsection{System Manual Procedure}

The KVR fusion system is a highly customized and user friendly system designed to enable ease of use by all. When the system is turned ON either via the AC supply mains or through the rechargeable backup battery, the initialization process of the device begins as shown in Figure 8. The initialization process basically displays on the LCD screen information such as the title of the project, the institution, registration number and so on. The next stage after this is the menu window which consists of a hash key "\#" and an asterisks key "*". These keys are used to access the next sub items embedded in the system.

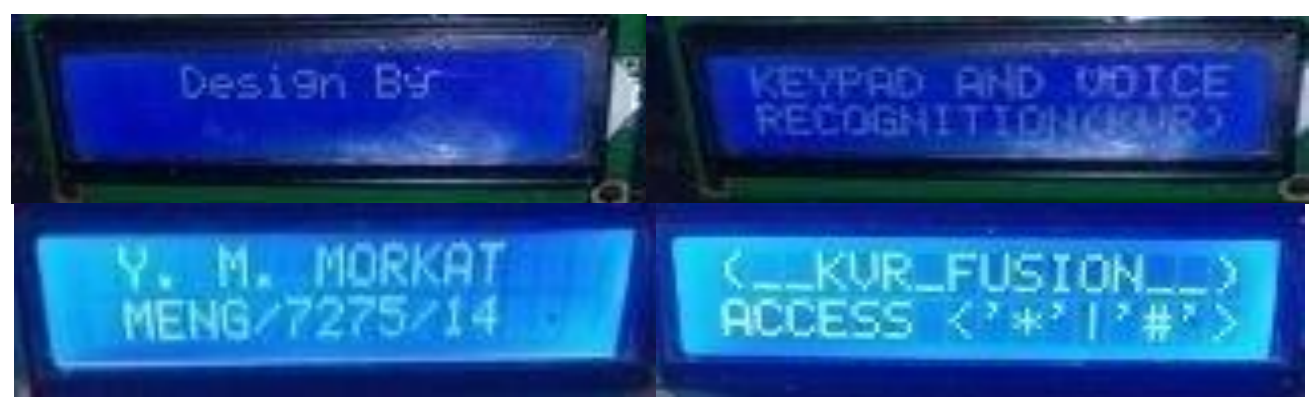

Figure 8. Initialization Process 


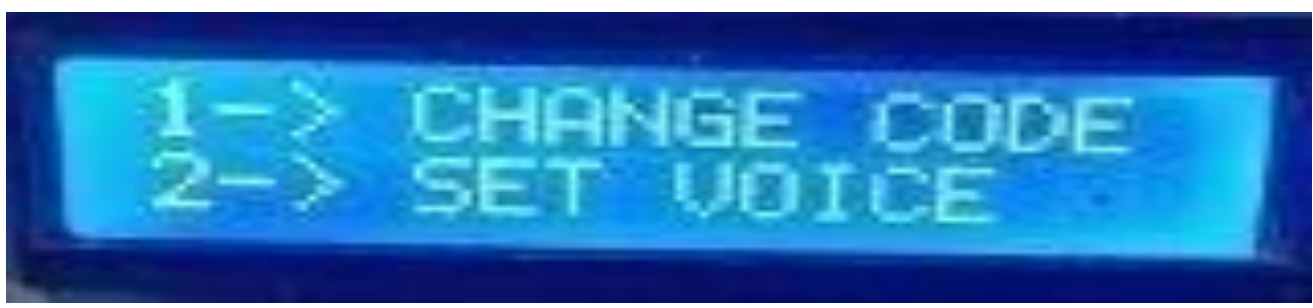

Figure 9. Menu of Hash “\#” Key

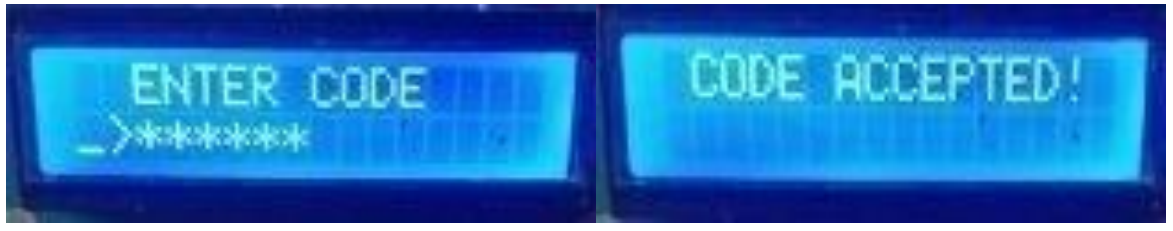

Figure 10. Change Code Command Accepted
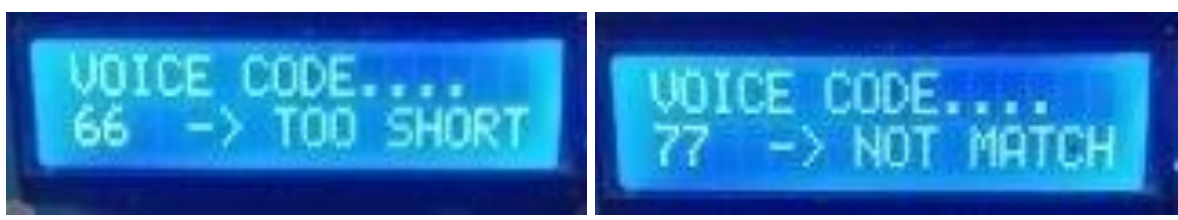

Figure 11. Error Code Display
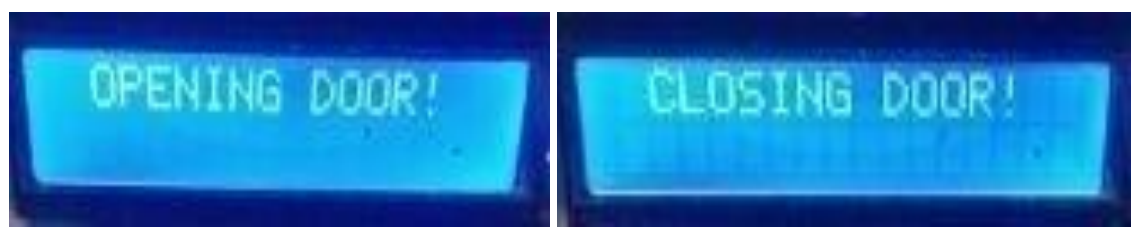

Figure 12. Door Opening and Closing

\subsubsection{The Hash “\#” Key}

The hash key, when selected, comes with its sub menu. These are change code and set voice options. Each of these options has an encryption that precedes the choice of the options. The numbers 1 and 2 are used to access either of the options. Figure 9 shows the menu of hash "\#” key.

\subsubsection{Change Code}

To change the six (6) digit encrypted code of the system which is ' 123456 ' to any six digits code of choice the number 1 is pressed from the keyboard as shown in Figure 10. The system will first demand the user to provide the current code which in this case can be the numbers 1 to 6 followed by a '\#' key. The \# key is used as an ENTER key at this stage while the * key is used as a delete key to enable the user correct a wrongly selected digit. When this is done the system will process and save the new security code in its EPROM. In order to return to the previous window the \# can be pressed again.

\subsubsection{Set Voice}

Selection of this option will enable the user to train and recognize voices. To achieve this, digit number 2 is pressed. As a way of authentication the system will again ask of the security code to be provided by the user. The system executes two tasks here: training of new voice command and recognition of the command train. 


\subsubsection{Training of System}

To train the system for a new voice entry, press the number 2 on the keyboard. The following will be displayed on the screen: "ENTER PATTERN NUMBER \& KEY \#". This means that the user should enter a valid pattern number which is a digit from 01,02 , to 40 follow by a \# key. The display 'SPEAK NOW' will prompt the user demanding a voice command to be fed through the microphone. When a word is spoken the green LED connected at the READY pin of the HM2007 will light ON and OFF to indicate that voice is accepted. Trained words can easily be changed by overwriting the original word.

\subsubsection{Recognition Process}

To confirm the word number previously stored press 1 under the set voice menu option. This will again require the six digits security code after which any voice command previously saved can be spoken. The system will process the command and display a match plus the word number of the spoken word on the LCD. If the spoken word is not contained in its library the system will display no match. After three wrong trials at any of the authentication stages the system is programmed to logout the user.

\subsubsection{The Asterisks “*”" Key}

This key is used for recognition of already saved words. It is one of the first menus we have access to immediately after initialization process. When a user has already trained the system and wishes to start using the system this key can be pressed for such purpose. When the key is pressed the system will demand the security code that has been saved after which the system will ask of a voice command depending on the task the user wishes to execute. This system is meant to open and close the door after some period of time.

\subsubsection{Error Codes}

When interfacing the external circuit through its data bus, the decoding circuit must recognize word numbers from error codes. The circuit is designed to recognize error codes 55, 66, and 77 and not to confuse them with word spaces 5, 6 and 7, as shown in Figure 11. These errors are predefined codes that are embedded in the chip but can be enabled by the program code.

\subsubsection{Inward Switch}

After the authentication and after users have been granted access there is no need for another validation procedure in order to exit the door. In order to bypass this process the system has an inward switch which when pressed enables users to go out of the building. The door opens and closes automatically when this switch is pressed as shown in Figure 12.

\subsection{Recognition Accuracy Test}

The first set of tests to be conducted is the recognition accuracy. This is the most important parameter in any voice recognition system because it tests the whole system from start to end. In some extreme cases the system can become unusable or even dangerous when the recognition accuracy percentage drops too low. The device has been trained with several words. For the purpose of this experiment the 'OPEN' and 'CLOSE' voice command saved in the memory locations 03 and 04 were used. To test the efficiency of the KVR system, the system was trained with 'userl' providing the voice command. It was then tested over 4 experimental trials by 3 users. User1 (male), User 2 (female) and User 3 (male) to recognize the previously trained voice commands and the following results were obtained: 
Table 1. Recognition Accuracy Test

\begin{tabular}{|c|c|c|c|c|c|c|c|c|c|c|c|c|c|}
\hline \multirow{2}{*}{$\begin{array}{l}\text { Load } \\
\text { Motor (Door) }\end{array}$} & \multirow[t]{2}{*}{ Command } & \multicolumn{4}{|c|}{ User1 } & \multicolumn{4}{|c|}{ User2 } & \multicolumn{4}{|c|}{ User3 } \\
\hline & & \multicolumn{4}{|c|}{ No. of Trials } & \multicolumn{4}{|c|}{ No. of Trials } & \multicolumn{4}{|c|}{ No. of Trials } \\
\hline & Open & $\sqrt{ }$ & $\sqrt{ }$ & $\sqrt{ }$ & $\sqrt{ }$ & $\sqrt{ }$ & $\sqrt{ }$ & $x$ & $\sqrt{ }$ & $\sqrt{ }$ & $\sqrt{ }$ & $x$ & $\sqrt{ }$ \\
\hline & Close & $\sqrt{ }$ & $\sqrt{ }$ & $\sqrt{ }$ & $\sqrt{ }$ & $x$ & $\sqrt{ }$ & $\sqrt{ }$ & $y$ & $x$ & $\sqrt{ }$ & $\sqrt{ }$ & $\sqrt{1}$ \\
\hline
\end{tabular}

Key: $\sqrt{ }$ (Matched); $\times($ Unmatched $)$

The percentage accuracy can be calculated from equation (1):

$$
\begin{aligned}
& \text { System Accuracy }=\frac{\text { Number of Match output }}{\text { Wumber of Experiment }} \times 100 \% \\
& \text { System accuracy of User } 1=\frac{8}{8} \times 100=100 \% \\
& \text { System accuracy of User2 }=\frac{5}{8} \times 100=62.5 \% \\
& \text { System accuracy of User3 }=\frac{6}{8} \times 100=75 \%
\end{aligned}
$$

Thus for the user training and operating the system, the success rate (system accuracy) was found to be $100 \%$ while for different users with different vocal patterns the success rates were found to be $62.5 \%$ and $75 \%$ respectively. A system that was trained by a particular gender has higher success rate when experimented by a different user of the same gender; this can be seen from the results of the test in Table 1 .

\subsection{Distance Limit between Speaker and Microphone}

The maximum allowable distance between a microphone and a user is important as it contributes to the accuracy or nature of result to be obtained while using the system. The distance between users with a microphone is shown in Table 2. As a conclusion, the matched and unmatched identity of a user depends largely on the distance between the user and the microphone. The environment and sensitivity of the type of microphone used also play a major role here.

Table 2. Distance Limit Test

\begin{tabular}{|l|l|l|}
\hline User & Microphone Distance & Matched/Unmatched Output \\
\hline User1 & $1.0 \mathrm{~cm}$ to $8.0 \mathrm{~cm}$ & Matched \\
\hline User2 & $8.0 \mathrm{~cm}$ to $16.0 \mathrm{~cm}$ & Matched \\
\hline User3 & $16.0 \mathrm{~cm}$ to $30.0 \mathrm{~cm}$ & Unmatched \\
\hline
\end{tabular}

\subsection{Training/Recognition in Noisy Environment}

The type of signal used for this experiment is music from a sound system. Music was used while training and recognizing the system at different distances as usedin the experiment above. Noise from music shows waveform with high intensity which is capable of causing unmatched output even with the user that trains the system. With noise at the background the allowable distance between the user and microphone varies as can be seen in Table 3 . 
Table 3. System Behaviour in Noisy Environment

\begin{tabular}{|l|l|l|}
\hline User & Microphone Distance & Matched/Unmatched Output \\
\hline User1 & $1.0 \mathrm{~cm}$ to $6.0 \mathrm{~cm}$ & Matched \\
\hline User2 & $6.0 \mathrm{~cm}$ to $16.0 \mathrm{~cm}$ & Unmatched \\
\hline User3 & $16.0 \mathrm{~cm}$ to $30.0 \mathrm{~cm}$ & Unmatched \\
\hline
\end{tabular}

\subsection{Distance Limit}

The distance between the user and the microphone in a noiseless environment should be between $1.0 \mathrm{~cm}$ to16 $\mathrm{cm}$ from the experiment conducted. This distance depends on the sensitivity of the microphone and the environmental condition at the time of the experiment. It was deduced that for a noisy environment the minimum and maximum acceptable distance between the user and microphone is greatly reduced due to the presence of noise from the background. The recommended distance under noisy condition between users and microphone is $1.0 \mathrm{~cm}$ to $6.0 \mathrm{~cm}$.

\section{Conclusion and Recommendations}

\subsection{Conclusion}

The system was built based on keypad and biometric concept for accessing a door. The biometrics used here is the human voice which can lock or unlock the door using voice command as the input of the system. The prototype of the keypad and voice recognition (KVR) fusion for security door system was successfully developed and the door can be locked or unlocked using the voice print while the keypad provides the security code for the first authentication process. The successful performance of the system was found to depend on the noise level of the environment. In a noiseless environment the distance at which the human voice is recognized by the system is measured to be up to $16 \mathrm{~cm}$ while in a noisy environment this distance is limited to $6 \mathrm{~cm}$. It was also discovered that the system was sensitive to the gender that provided the voice input for training.

\subsection{Recommendations}

The system was functional as expected, but the performance of the system at the DSP chip was moderately efficient as the system at the recognition level during the second security verification phase tends to recognize same command spoken by a different user who did not train the system. Even though the tendency for this recognition is very low it provides a little drawback for the research. To complement for this present limitation that comes with the voice recognition chip the system was integrated with a security code to optimize the security level. For future work, some recommendations have been listed to solve the problems and remove the limitations in order to enhance the performance.

i. Use connected or continuous words to train and recognize the system. This can help reduce, to a great extent, different user recognition accuracy.

ii. The voice recognition chip can be connected in cascaded form so that larger memory can be used.

iii. Other forms of biometrics technology like the fingerprint, iris print and facial synthesis with a GSM module can be fused to the system to optimize the security architecture of the system if it is to be used for security purpose. 


\title{
References
}

[1] Organization for Economic Co-Operation and Development (OECD), "Making Poverty Reduction Work", https://www.oecd.org/development/34839878.pdf. (Accessed 10 ${ }^{\text {th }}$ November 2015).

[2] S. H. K. Yahya, "Speaker Independent Voice Recognition Calculator", Contemporary Engineering Sciences, vol. 5, no. 3, (2012), pp. 119-125.

[3] M. Faundez-Zanuy, J. Fierrez-Aguilar, J. Ortega-Garcia and J. Gonzalez-Rodriguez, "Multimodal Biometric Databases", IEEE Aerospace and Electronic Systems Magazine, vol. 21, no. 9, (2006), pp. 2937.

[4] P. T. Krishna and K. D. Kranti, "Voice Controlled Autonomous Wheelchair", Proceedings of National Conference on Knowledge, Innovation in Technology and Engineering (NCKITE), Raipur, Chhattisgarh, India, (2015) April 10-11.

[5] HM2007 Speech Recognition, https://www.imagesco.com/speech/HM2007.pdf. (Accessed 4th June, 2015).

[6] W. A. Wahyudi and M. Syazilawati, "Intelligent Voice-Based Door Access Control System Using Adaptive-Network Based Fuzzy Interference Systems (ANFIS) for Building Security", Journal of Computer Science, vol. 3, no. 5, (2007), pp. 274-279.

[7] S. T. Dhawan and S. Aditi, "Voice Recognition Wireless Home Automation System Based on Zigbee", IOSR Journal of Electronic and Communication Engineering (IOSR-JECE), vol. 6, no. 1, (2013), pp. 65-75.

[8] A. M. Omar, T. H. Rasha and T. Nicolae, "Access Control Using Biometrics Features with Arduino Galileo", International Journal of Advanced Research in Computer Science and Software Engineering, vol. 4, no. 8, (2014), pp. 134-140.

[9] CCS Compiler, https://sourceforge.net/directory/os:windows/?q=ccs\%20c\%20compiler\%20for\%20pic (Accessed 15 $5^{\text {th }}$ May, 2015)

[10] PIC16F87XA Data Sheet, http://ww1.microchip.com/downloads/en/DeviceDoc/39582b.pdf (Accessed $4^{\text {th }}$ June, 2015).

[11] L293D DataSheet,https://www.ti.com/lit/ds/symlink/1293.pdf. (Accessed $4^{\text {th }}$ June, 2015).

[12] Pic Kit2 programmer, https://www.pickit2.software.informer.com/2.6/. (Accessed $4^{\text {th }}$ June, 2015).

\begin{abstract}
Authors
Morkat M. Yilwatda, holds the degree of Bachelor of Engineering in Electrical and Electronics Engineering. He is currently pursuing the Masters degree in the same discipline at the Department of Electrical and Electronics Engineering, University of Agriculture, Makurdi, Nigeria where he is also a graduate assistant.
\end{abstract}

Jonathan A. Enokela, is an Associate Professor in the Department of Electrical and Electronics Engineering, University of Agriculture, Makurdi, Nigeria. He has taught various aspects of analog and digital systems design to both the undergraduate and postgraduate students. He is a registered engineer with the Council for Regulation of Engineering in Nigeria (COREN) and has a wide range of practical experiences. His research interests include embedded systems design and applications.

Nentawe Y. Goshwe, is a Senior Lecturer in the Department of Electrical and Electronics Engineering, University of Agriculture, Makurdi, Nigeria. He has several years of teaching experience in electronics at the undergraduate and postgraduate levels. $\mathrm{He}$ is a registered engineer with the Council for Regulation of Engineering in Nigeria (COREN) and has a wide range of practical experiences. His research interests include power electronics and automotive systems. 
International Journal of Security and Its Applications

Vol. 11, No. 4 (2017) 